\title{
Apuntes para la historia de la gerontología en Yucatán, México.
}

\author{
Historia de la Medicina
}

Orlando I. Piña-Basulto.

Universidad Mesoamericana de San Agustín, Mérida, Yucatán, México.

\section{RESUMEN.}

Ante el evidente crecimiento de la población mayor de 60 años y la escasez de personal idóneo para atender sus múltiples demandas a corto y mediano plazo, un grupo de profesionales de diferentes disciplinas e instituciones, decidieron organizarse en Yucatán, México, en 1999, bajo los auspicios de la Facultad de Medicina de la Universidad Autónoma de Yucatán y de la Rectoría de la Universidad Mesoamericana de San Agustín, en una Comisión para la elaboración del plan de estudios de la Licenciatura en Gerontología. Se hace un bosquejo del panorama local gerontológico y se describe la constitución subsecuente, en el año 2000, del Comité Interinstitucional Para el Estudio Gerontológico en Yucatán (CIPEGY) que dio origen a la impartición de los primeros diplomados universitarios en gerontología y a la difusión de una cultura sobre el problema del envejecimiento y de propuestas para afrontarlo. Se mencionan los nombres de los promotores iniciales de la profesionalización de la gerontología en Yucatán.
Palabras clave: Historia de la medicina, gerontología, Yucatán México.

(Rev Biomed 2003; 14:205-210)

\section{SUMMARY.}

Notes on the history of gerontology in Yucatan, Mexico.

In view of the evident increase in the population over 60 years old, and the lack of properly trained personnel to take care of their multiple short and longterm needs, a group of professionals from various disciplines and institutions decided to organize the development of a curricula on gerontolgy at the undergraduate level. A comission was formed for this purpose under the sponsorship of the Medicine School of the Universidad Autónoma de Yucatán (UADY) and the Universidad Mesoamericana de San Agustín (UMSA). An overview of the local situation regarding gerontology is presented, and a description of the creation in the year 2000, of the Interinstitutional Committee on Gerontologic studies of Yucatan (CIPEGY) is given. The names of the initial promoters

Solicitud de sobretiros: M.C. Orlando I. Piña-Basulto. Centro de Especialidades Médicas (CEM), Calle 60 \# 329-B x Av. Colon, C.P. 97000, Mérida, Yucatán, México.Tels.9201651 y 9256164. Correo electrónico: dr_opb@yahoo.com.mx

Recibido el 17/Septiembre/2003. Aceptado para publicación 26/Septiembre/2003.

Este artículo está disponible en http://www.uady.mx/sitios/biomedic/revbiomed/pdf/rb031438.pdf

Vol.14/No.3/Julio-Septiembre, 2003 


\section{OI Piña-Basulto.}

in Yucatan of gerontology as a profession are mentioned. (Rev Biomed 2003; 14:205-210)

Key words: History of medicine, gerontology, Yucatan, Mexico.

\section{Introducción.}

El crecimiento poblacional, tanto a nivel mundial como regional, implica un aumento considerable de personas mayores de 60 años $(1,2)$. Las estimaciones efectuadas por la ONU señalan el notable incremento en este grupo de edad al final de la primera década de este siglo XXI, pero sobre todo en el año 2025 $(3,4)$. Estos datos sociodemográficos representan un desafío a la ciencia, a la sociedad y a los gobiernos. A la primera, porque se requiere un mayor conocimiento de los procesos del envejecimiento humano en sus vertientes biológica, psicológica y social. A la sociedad, porque deberá tomar conciencia del fenómeno, su realidad y sus consecuencias. A los gobiernos, porque serán los responsables de diseñar y promover nuevas políticas de atención a este grupo poblacional (5-8).

Una de las herramientas prioritarias para satisfacer la demanda cada día más creciente de este sector, es la gerontología, ciencia hermanada a la geriatría, pero con perfil propio, ya que la geriatría se ocupa de la atención del anciano enfermo, en tanto que la gerontología rebasa el campo clínicomédico $(9,10)$.

Existen diferentes definiciones de gerontología. Entre otras: "Ciencia que estudia el proceso del envejecimiento desde los puntos de vista biológico, psicológico y social."; "estudio de los problemas y fenómenos propios de la vejez"; "disciplina aplicada del área social y educativa que considera a los adultos mayores protagonistas de su propia existencia y capaces de seguir viviendo dignamente y de dirigir su propia vida" $(9,11,12)$.

Desde el año 1990, la ONU designó el 1 de octubre como "Día internacional de la Personas Mayores" con el fin de concientizar a la población, sobre los problemas y necesidades de las personas de mayor edad. Ya antes, en 1982, en Viena, se había realizado la Primera Asamblea Mundial sobre Envejecimiento, auspiciada por la ONU. En 1999, el Papa Juan Pablo II, así como la ONU, declaran el "Año Mundial del Anciano", enfatizando que los problemas y nuevas situaciones debidas al envejecimiento poblacional son de interés para la Humanidad. A partir de la "Segunda Asamblea Mundial Sobre el Envejecimiento", celebrada en Madrid del 8 al 12 de abril de 2000, muchos países y organizaciones han encaminado serios esfuerzos para establecer una serie de políticas y actividades destinadas a mejorar la calidad de vida del adulto mayor, así como para atender las demandas en salud, educativas y económicas que traen aparejados estos cambios demográficos $(4,13)$.

\section{Hacia el enfoque del problema.}

Estos brevísimos conceptos, que podrían ahora parecer muy obvios, no lo eran tanto a principios de 1999, cuando decidimos adentrarnos al mundo de la gerontología en Yucatán. En esa época cuando yo finalizaba el primer año como Rector de la Universidad Mesoamericana de San Agustín (UMSA), ubicada en la ciudad de Mérida, Yucatán, se planeó diversificar la oferta académica de la región. Al enterarnos que las licenciaturas de Médico Cirujano y Cirujano Dentista estaban saturadas y no se recomendaba la apertura de nuevos programas, decidimos explorar en el campo de la gerontología. Acudimos al Rector de la Universidad Autónoma de Yucatán (UADY), para proponerle un trabajo conjunto. Él nos envió a la Facultad de Medicina, donde hacía unos meses se había iniciado un proyecto sobre Gerontología, coordinado por el Dr. Hernando Alberto Puga Navarrete y por el Dr. René Peniche Campos. Unimos esfuerzos y voluntades retomando el proyecto de licenciatura en gerontología, con el fin de obtener la aprobación del $\mathrm{H}$. Consejo Universitario de la UADY, y que después la UMSA incorporara dicha carrera a sus planes de estudio. Integramos un grupo de trabajo que denominamos "Comisión Interinstitucional y Multidisciplinaria para la Elaboración del Plan de Estudios de la Licenciatura en Gerontología”, el cual comenzó a funcionar

\section{Revista Biomédica}


Historia de la gerontología en Yucatán, México.

oficialmente el martes 23 de febrero de 1999 (Peniche-Campos R. Archivo de la Secretaría Académica de la Facultad de Medicina de la UADY;1999). Además del Dr. Puga, el Dr. Peniche y el Dr. Piña, aceptaron colaborar: el Dr. José Bolio García, Médico Geriatra y profesor de las Facultades de Medicina y Enfermería de la UADY; el Psicólogo Raúl Burgos Fajardo, maestro de la Facultad de Educación y responsable del Departamento Técnicopedagógico de la UMSA; la Cirujana Dentista y Gerontóloga Elsa Gamboa Medina, funcionaria del INSEN-Yucatán; el Psicólogo Rodolfo González Huerta, Director de Desarrollo Educativo Integral, la Enfermera Rosa Otilia Güemes Medina, maestra de la Facultad de Enfermería; la Antropóloga Cristina Leyrana Alcocer; el Gerontólogo Fernando Magaña Escamilla (Jefe de Enseñanza y titular de los cursos monográficos sobre gerontología, del Hospital Benito Juárez del IMSS); el Abog. Andrés Novelo Álvarez (Titular de la Procuraduría de Defensa del Anciano en Yucatán); el Dr. Miguel Jaime Pat Chuc, Médico Geriatra y Profesor de la Preparatoria de la UADY; las Enfermeras Guadalupe Quintal Alpuche y Marta López Osalde, de Desarrollo Educativo Integral (DEI); y Leticia Molina Cámara, Subdirectora del Centro Gerontológico "La tercera edad"; el Br. Ricardo Alpuche Mézquita (Delegado del INSEN) y la Dra. Irene Rivas Garrido (Directora del Instituto Geriátrico Mexicano, A.C. y una de las pioneras en la atención geriátrica hospitalaria en Yucatán). A este grupo se nos agregaban ocasionalmente otros profesionales interesados en la cultura gerontológica y profesores de la Facultad de Medicina o de la UMSA, entre otros, la Dra. María Luisa Rojas Bolaños, el Abogado Gabriel Brito Alonzo, la Lic. en Nutrición Reyna Bojórquez Cruz, el Dr. Jorge Cimé Martín y el Dr. Raúl Mena López, profesor titular del CINVESTAV del IPN.

\section{Las primeras conclusiones.}

Como resultado de estas sesiones de trabajo, se concluyó, en primera instancia, que no existía en Yucatán, ni en la mayoría de las entidades federativas mexicanas un conocimiento adecuado del proceso del envejecimiento individual y poblacional, ni tampoco existía conciencia en la población de los retos y oportunidades que este envejecimiento traería consigo. Había por supuesto personas interesadas en la gerontología, pero eran muy pocas y su enorme esfuerzo pasaba desapercibido o no era comprendido en toda su valía. También era cierto que en diversas Instituciones, como en el Instituto Mexicano del Seguro Social (14), el Instituto de Seguridad Social al Servicio de los Trabajadores del Estado, la Secretaría de Salud, el Sistema de Desarrollo Integral de la Familia, el Ayuntamiento de Mérida, el Instituto Nacional de la Senectud, y el Instituto de Seguridad Social de los Trabajadores del Estado de Yucatán se tenían programas para la tercera edad, pero generalmente desarticulados entre sí y con limitaciones, dados sus bajos presupuestos para este rubro y ejecutados por personal muy bien intencionado, pero en pocos casos profesionalizado en esta área.

Los asilos y casas-hogar se mantenían principalmente por aportaciones y donaciones altruistas, siendo la Iglesia Católica, a través de alguna congregación, quien contribuía principalmente a paliar el problema, sobre todo de los ancianos desamparados o menos favorecidos. La Iglesia Presbiteriana y algunas instituciones particulares laicas también aportaban, pero más modestamente. En el año 2000, sólo ejercían tres médicos geriatras en todo el Estado de Yucatán. A la fecha, se ha incorporado uno más. A principios de ese año, tanto la UMSA, como la Facultad de Medicina de la UADY, hicieron sendas encuestas sobre gerontología entre jóvenes de nivel bachillerato y en profesionales, tanto del área de la salud como de otras áreas. Los resultados de ambas fueron desoladores: ni en estos niveles pudieron definir claramente la diferencia entre geriatría y gerontología. Es más, muchos encuestados no sabían qué es un gerontólogo o un especialista en gerontología y mucho menos estaban concientes del envejecimiento poblacional, proceso que nos afectará a todos, sin distinción (Piña-Basulto O. Archivo de la Rectoría de la Universidad Mesoamericana de San Agustín. 19992003). 


\section{OI Piña-Basulto.}

\section{La gerontología y la actividad académica.}

En relación con programas académicos sobre gerontología, en toda la República Mexicana, a nivel licenciatura, solamente existía uno y a prueba, en el Colegio Holandés de la Ciudad de México, D.F. (que, por cierto, dejó de impartirse por razones administrativas). A nivel de postgrado solamente había posibilidad de entrenarse en la Ciudad de México, D.F. o en el extranjero. A nivel local, se habían impartido algunos cursos o talleres de tipo monográfico y de pocas horas de duración; en el local de la empresa Desarrollo Educativo Integral, las enfermeras mencionadas anteriormente habían impartido un Diplomado no universitario de 80 horas. En el Centro de Bachillerato Técnico Industrial No. 112 estaban también abriendo brecha en el campo gerontológico, al impartir el bachillerato técnico en gericultura, con pocos alumnos.

Ante este panorama gerontológico, el sentir fue que no deberíamos limitarnos exclusivamente a trabajar sobre la licenciatura en gerontología, sino a unir ideales y ánimos para ampliar nuestros objetivos. Así, se creó una agrupación civil que denominamos "Comité Interinstitucional para el Estudio Gerontológico en Yucatán" (CIPEGY), el 21 de noviembre de 2000, en la Facultad de Medicina de la UADY. Los fines y objetivos de la misma fueron:

1.- Fomentar una cultura de atención integral y de calidad para la población adulta mayor.

2.- Promover la investigación sobre el envejecimiento y difundir los resultados de las mismas.

3.- Promover la capacitación y formación de recursos humanos para la atención de esta población

4.- Apoyar las acciones para la protección de la población mayor de 60 años de edad

5.- Colaborar en la formación, regularización, normatividad y vigilancia de los servicios asistenciales para este grupo poblacional.

Se eligió la primera directiva quedando integrada de la siguiente forma:

Presidente: Dr. Alvaro H. Puga Navarrete

Secretario: Dr. Orlando I. Piña Basulto

Vocal I Coord. Planeación: Lic. Rodolfo González Huerta
Vocal II Coord. Capacitación: Enf. Guadalupe Quintal Alpuche.

(Posteriormente se designaría Tesorero al Dr. Fernando Magaña Escamilla.)

Esta directiva entraría en funciones de inmediato y por dos años. Una vez firmada el Acta Constitutiva se registró ante el Escribano Público No. 10 de la Ciudad de Mérida, Abogado Antonio Kú Pérez.

El Comité empezó a trabajar concientizando a la población en la cultura del envejecimiento y dando difusión a la nueva Ley para la Protección Social de las Personas en Edad Senescente del Estado de Yucatán, aprobada por el H. Congreso Estatal (15). Además de las conferencias públicas que se llevaron a cabo, se logró reunir a más gentes interesadas en la gerontología y que trabajaban aisladamente. Del trabajo de este grupo salió la decisión de impartir en la UMSA, el Primer Diplomado Universitario en Gerontología, de 160 horas, y con reconocimiento de la UMSA y de la UADY. Éste se realizó en el ciclo escolar 2001-2002, siendo el Profesor Titular el Dr. Fernando Magaña Escamilla.

La propuesta del plan de estudios de la Licenciatura en Gerontología de la Facultad de Medicina de la UADY, no fue aprobada por diversas razones. Entre otras, según el SubComité de Educación del Comité Nacional de Atención al Envejecimiento de la SSA y de la comisión evaluadora de la propuesta respectiva, porque no estaba bien fundamentada, se orientaba más al campo médico, se confundía el perfil de egreso con otras profesiones (por ejemplo, con el de la Licenciatura en Rehabilitación) y por la oposición de algunas autoridades que no comprendían que se trataba de crear una nueva profesión, con formación multidisciplinaria, pero dedicada a un sector de la población que se incrementaría en los próximos años y que demandará una atención profesionalizada. No se trataba únicamente de procurar la capacitación profesional a nivel técnico o de postgrado, sino insistíamos en el deber de profesionalizar la atención del problema del envejecimiento a nivel licenciatura. Por lo anterior se decidió en el seno del CIPEGY hacer las correcciones pertinentes (aunque seguimos

\section{Revista Biomédica}




\section{Historia de la gerontología en Yucatán, México.}

considerando un enfoque integral del egresado, la mayor carga sería hacia lo social y administrativo) y continuar obteniendo mayor información para justificarla y fundamentarla, contando con el apoyo de la UMSA a través de su Rector, y de la C.D. y gerontóloga Elsa Gamboa Medina, para lograr la aprobación de esta carrera. A fines de agosto de 2003 la Secretaría de Educación del Gobierno del Estado de Yucatán, aprobó el programa, por lo que al iniciarse, será el primero con nivel de licenciatura, en todo el país.

\section{Colofón.}

Actualmente las cosas han cambiado para bien. Prácticamente el CIPEGY ya no tiene razón de ser, ya que tanto el gobierno federal como el local han dado mayor importancia al problema de la vejez. Se ha constituído, a semejanza del nacional, un Comité Estatal para la Atención del Envejecimiento y del cual formamos parte no solamente muchos de los que iniciamos estos trabajos, sino representantes oficiales de instituciones públicas y privadas. Tanto la UMSA como la UADY forman parte de este grupo y en nuestras Instituciones hemos sembrado la semilla de una cultura de un envejecimiento digno y con mejor calidad de vida. En estos meses se realizará un trabajo de equipo, coordinado por la Facultad de Medicina y propuesto por la OPS sobre el proyecto Salud y Bienestar en el Envejecimiento (SABE) $(16,17)$. En los últimos días de septiembre del presente año finalizaráel II Diplomado Universitario en Gerontología (UADY-UMSA) y hemos comenzado los trabajos para una Maestría en Gerontología y un Programa Universitario o Aulas para la Tercera Edad. Sabemos que cada día va a haber más y mejor gente interesada, preparada y dedicada a la gerontología en Yucatán, México.

\section{REFERENCIAS.}

1.- Kinsella K. Dimensiones demográficas y de salud en América Latina y el Caribe. En: Anzola-Pérez E. Editor. La atención de los adultos mayores: un desafío para los años noventa. Washington: Organización Panamericana de la Salud; 1994. p. 3-18.
2.- INEGI. La Tercera Edad en México. XI Censo General de Población y Vivienda. $2^{\mathrm{a}}$ ed. Aguascalientes:INEGI;1993.

3.- ONU. Population Ageing Wall Chart. División de Población, Departamento de Asuntos Sociales y Económicos. New York: ONU; 1999.

4.- OPS/OMS. Plan de acción en salud y envejecimiento. Los adultos mayores en las Américas. División de Promoción y protección de la salud. Unidad de envejecimiento y salud. Washington: Organización Panamericana de la Salud; 1999.

5.- Kalache A, Coombes Y. Population aging and care of the elderly in Latin America and the Caribbean. Rev Clin Gerontol 1995; 5:347-55.

6.- Gónzalez-Molina J. La OPS/OMS y la atención del adulto mayor. Gerontología y Geriatría 1999; 1:6-10.

7.- CONAPO. Envejecimiento demográfico de México:Retos y perspectivas. $1^{a}$ ed. Senado de la República, Comisión de Población y Desarrollo; Cámara de Diputados. México: H.Congreso de la Unión;1999.

8.- Gómez de León J, Rabell C. La población de México.Tendencia y perspectiva sociodemográfica hacia el siglo XXI. México: CONAPO, Fondo de Cultura Económica; 2000.

9.- Prieto-Ramos O, Vega-García E. Temas de gerontología. La Habana: Editorial Científico-Técnica; 1997.

10.- Lara-Rodriguez M. El adulto mayor y sus problemas de salud, nuevo reto para los servicios institucionales. En: Problemas y programas del adulto mayor. Conferencia Interamericana de Seguridad Social ( CIESS). México;1997. p. $137-51$

11.- Laforest J. Introducción a la gerontología. México: Herder; 2002.

12.- Moragas R. Gerontología Social. Colección Albor.México: Herder; 2002.

13.- INAPLEN. Conclusiones del Foro de Consulta Nacional sobre el Envejecimiento en México. México: INAPLENSEDESOL; 2001.

14.- Lozano-Dávila M, Trujillo Z, Chavez J, Pérez-Ramos M. Diplomado de Gerontología con enfoque de riesgo a distancia,experiencia del IMSS. Gerontología y Geriatría 2000; 3: 13-17. 


\section{OI Piña-Basulto.}

15.- Gobierno del Estado. Ley para la protección social de las personas en edad senescente del Estado de Yucatán. Decreto No. 214. Mérida: Diario Oficial del Gobierno Libre y Soberano del Estado de Yucatán. agosto 16,1999.

16.- OPS. Proyecto SABE: salud, bienestar y envejecimiento en América Latina y el Caribe. Coordinación de Investigaciones, División de Salud y Desarrollo Humano, Washington D.C. ; Organización Panamericana de la Salud; 1999.

17.- Health Canada/ OPS/ SSA. Guía para el desarrollo de un sistema integral de apoyo para el envejecimiento activo. Washington, D.C. Organización Panamericana de la Salud; 2002.

Fe de erratas:

En el artículo:

Jiménez-Cruz A, Bacardí-Gascón M, Rosales-Garay P, Herrera-Espinosa J, Willis WO. A culturally sensitive tool for Mexican people with diabetes: "La manzana de la salud". Rev Biomed 2003; 14:51-59.

El último nombre de los autores dice:

Willis O. Willis.

y debe decir:

Winnie O. Willis. 